\title{
Numerical Analysis of a Bi-Directional Synthetic Jet for Active Flow Control
}

\author{
Rodrigo Fisher ${ }^{*}$, Takafumi Nishino ${ }^{\dagger}$, Mark Savill ${ }^{+}$ \\ Cranfield University, Cranfield, Bedfordshire, MK430AL, United Kingdom
}

\section{Introduction}

$\mathrm{B}$ OUNDARY layer separation control by the action of Synthetic Jets has been widely studied for more than a decade; its success as an active flow control device has been extensively reported by several authors ${ }^{1,2,3,4,5,6}$. The parameters that modify the behavior of Synthetic Jets include the cavity shape, the diameter of the exit, and the frequency and amplitude of the excitation, while the formation and evolution of Synthetic Jets strongly depend on the Strouhal and Reynolds numbers ${ }^{7}$. It is also known that good flow control performance is achieved at relatively high oscillation frequencies ${ }^{6}$.

In 2004, several institutions around the world worked with NASA to study the capability of Reynolds-averaged Navier-Stokes (RANS) simulations using three validation cases with Synthetic Jets. Rumsey ${ }^{8}$, who compiled the results, reported that although some details of the velocity field close to the jet exit were not captured by the numerical analyses, the general trend was reproduced with reasonably good accuracy compared with experiments. Four years later, the outcomes of this international work were published including some new results obtained after the workshop 9 . One of the main conclusions was that the Spalart-Almaras (S-A) and $k-\omega$ Shear-Stress-Transport (SST) models captured reasonably well many features of interest.

In one of the three validation cases used for the workshop, the effect of a Synthetic Jet was modeled over a wallmounted hump at $65 \%$ of the chord. This case, known as "NASA 2D Hump", has been used for many further validation studies, e.g. Gan et $\mathrm{al}^{10}$, who investigated a simple suction state, Greenblatt ${ }^{11}$, who performed a number of experimental analyses, and Rumsey ${ }^{12}$, who addressed the oscillatory flow behavior and compiled the work presented in the workshop ${ }^{8}$. The NASA 2D Hump has been found to be useful for validation of numerical analyses of

\footnotetext{
${ }^{*}$ Ph.D Candidate, School of Engineering, j.r.fisherfernandez@cranfield.ac.uk, AIAA Member.

'Lecturer, Centre for Offshore Renewable Energy Engineering, t.nishino@cranfield.ac.uk.

* Professor, School of Aerospace, Transport and Manufacturing, mark.savill@cranfield.ac.uk,AIAA Senior Member.
} 
Synthetic Jets because of its geometrical simplicity and well-documented range of results. Many different configurations of Synthetic Jets have been studied in the past. Also, new types of jet flow actuators have recently been developed to achieve oscillatory or sweeping jet blowing ${ }^{13,14}$; however, these new actuators require net mass flow and are therefore different from common Synthetic Jets.

In the present work, we numerically test an alternative novel active flow control concept, called Bi-Directional Synthetic Jet (BDSJ), using RANS simulations of flow over the NASA 2D Hump model. The term Bi-Directional expresses that the intake direction of the jet is different from the outflow direction, so the Coandă effect can be induced by ejecting the flow close to parallel to the surface, while the intake can still efficiently entrains the flow upstream to keep the boundary layer attached. A sketch of the Bi-Directional Synthetic Jet is presented in Fig. 1. The effects of the Bi-Directional Synthetic Jet are simulated numerically by prescribing the profile and direction of oscillatory flow at the jet exit boundary. The actual device, details of which will be investigated in future studies, may involve a rapidly oscillating magnetic membrane and an internal tube connecting two jet holes to entrain air into one hole and eject it from the other hole, changing the inflow and outflow directions of the jet. In the present study, however, we consider only one jet hole for simplicity.

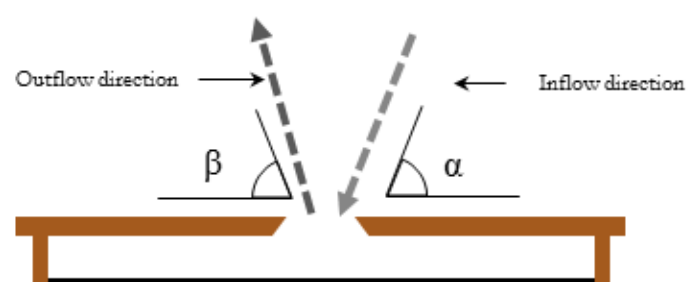

Fig. 1 Conceptual drawing of a Bi-Directional Synthetic Jet.

\section{Numerical Model}

\section{Computational domain}

The geometry of the hump emulates the upper surface of a $20 \%$ thick Glauert-Goldschmied airfoil. The computational domain mimics the test section of the experiments ${ }^{15}$, where the body was mounted between two glass endplates, and both leading and trailing edges matched smoothly the wind tunnel splitter-plate. The dimensions of the computational test section are $382 \mathrm{~mm}$ high and 3,531 mm long. The model leading-edge is placed at 4.61 chord lengths $(1935 \mathrm{~mm})$ from the inlet, and the length of the hump is $420 \mathrm{~mm}$, which has a $1.8 \mathrm{~mm}$ jet slot at $65 \%$ of the 
chord. The 2-D computational domain, together with the final mesh employed in this study, is shown in Fig. 2. The details of a grid convergence study will be described later in section II.5.

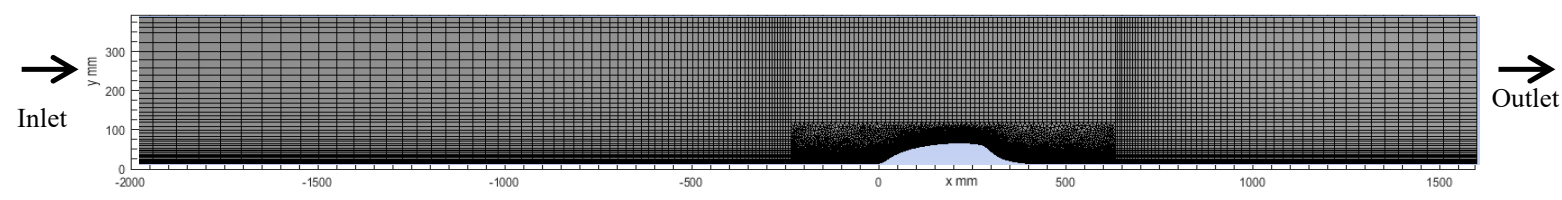

a) Entire domain

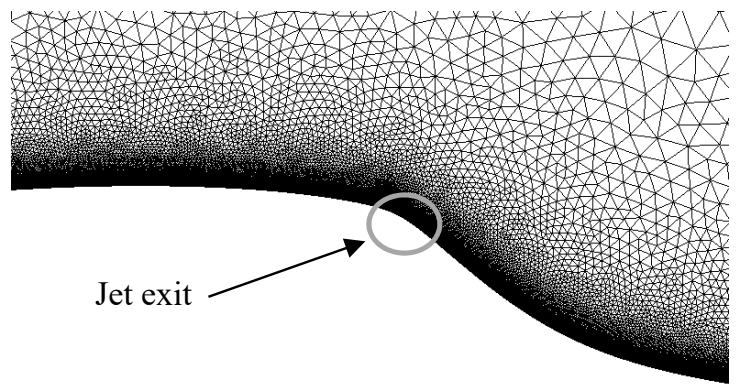

b) Over the hump

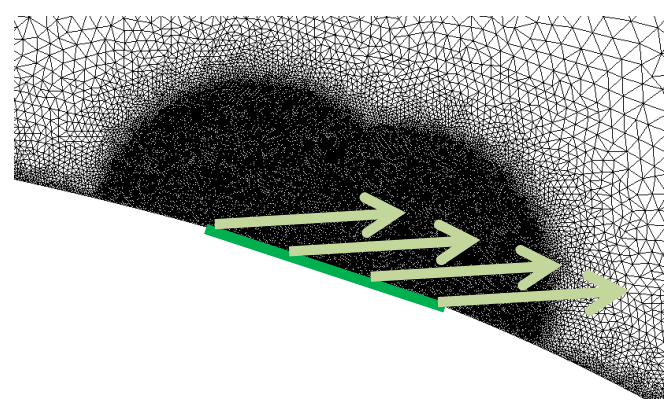

c) Around the jet exit.

Fig. 2 Discretized computational domain

\section{Flow solver and parameters}

The flow solver used in this study, ANSYS FLUENT v14, employs a control volume method to discretize the incompressible Reynolds-averaged Navier-Stokes (RANS) equations with second order accuracy into a series of algebraic equations that are numerically solved. The SIMPLE algorithm is used for pressure-velocity coupling for steady state calculations, while the PISO algorithm is used for transient calculations ${ }^{16}$ with a second-order fully implicit scheme for the time integration.

The working fluid is air with a viscosity of $1.84 \times 10^{-5} \mathrm{~kg} / \mathrm{ms}$ and a density of $1.185 \mathrm{~kg} / \mathrm{m}^{3}$, giving a Reynolds Number (based on the hump chord length) of approximately one million. The reference pressure is set at 101,325 Pa at the outlet of the domain. For all analyses presented in this paper, unless explicitly stated, the inlet velocity is $U_{\infty}=$ $34.6 \mathrm{~m} / \mathrm{s}$ (Mach 0.1 ), normal to the boundary, the turbulent intensity at the inlet is $5 \%$ and the turbulent length scale $1 \mathrm{~mm}$. Zero streamwise-gradient conditions are given at the outlet. The no-slip conditions are employed at the top and bottom walls (including the hump surface). Each computation is initiated with a uniform velocity $(u, v)=$ $\left(U_{\infty}, 0\right)$ given to the entire computational domain. 


\section{Turbulence model selection}

Although it is known that RANS turbulence models cannot predict quantitatively correctly the reattachment point downstream of the hump, they still tend to give qualitatively correct solutions that are sufficient for comparative studies. To find a trend in parametric studies, RANS simulations are still the most appropriate approach as large eddy simulations (LES) and detached eddy simulations (DES) would require prohibitive computational resources to solve a large number of cases, even though there are recent efforts on using wall-modeled LES to overcome such difficulties ${ }^{17}$. A summary of earlier studies using RANS turbulence models for this type of flow is given in Table 1.

Table 1 Turbulence models used by different authors.

\begin{tabular}{|c|c|c|}
\hline Studied by & Model used & Synthetic Jet over a \\
\hline Krishnan et al.(p.145) ${ }^{18}$ & S-A \& SST & 2D\&3D/Hump \\
\hline Morgan et al. (p.151) & Realizable $k$ - $\varepsilon$ & 2D\&3D/Hump \\
\hline Karthikeyan et al. (p.163) & S-A & 2D\&3D/Hump \\
\hline Rumsey ${ }^{19}$ & $\mathrm{~S}-\mathrm{A}, \mathrm{SST}, \& \mathrm{EASM}-\mathrm{k} \omega^{\S}$ & 2D/Hump \\
\hline Katam et al. (p.175) & SST & 2D/Hump \\
\hline Viken. et al. ${ }^{20}$ & S-A & 2D/Hump \\
\hline Balakumar (p.201) & SST & 2D/Hump \\
\hline Laouedj et al ${ }^{21}$ & SST & 3D/Flat Plate \\
\hline Lyons et al. ${ }^{22}$ & S-A & 3D/Hump \\
\hline Kim et al. ${ }^{23}$ & SST & 3D/Flat Plate \\
\hline He et al. ${ }^{24}$ & $k-\varepsilon, \mathrm{SST}, \mathrm{S}-\mathrm{A}$ & 2D/Hump \\
\hline Bettini \& Cravero $^{25}$ & RNG $k-\varepsilon^{* *}$ & 2D/Hump \\
\hline
\end{tabular}

In the present study, the Spalart-Allmaras, the Realizable $k-\varepsilon$, and $k-\omega$ SST turbulence models are used as they have shown to be reasonably reliable for this type of flow.

\section{Modeling of the Synthetic Jet}

The Synthetic Jet is modeled by manipulating the velocity boundary conditions across the jet exit (located at $x / c=0.65$ ), similarly to the study by Viken et al. ${ }^{20}$ Eleven different angles are considered for the inflow/outflow directions of the jet, as described in Fig. 3. It has been reported that different cavity shapes do not affect significantly the flow characteristics at the jet exit ${ }^{26}$; therefore the actual cavity is not modeled in this study.

\footnotetext{
${ }^{\S}$ Explicit Algebraic Stress Model in $k-\omega$ form.

${ }^{* *}$ Renormalization Group Model in $k-\varepsilon$ form.
} 


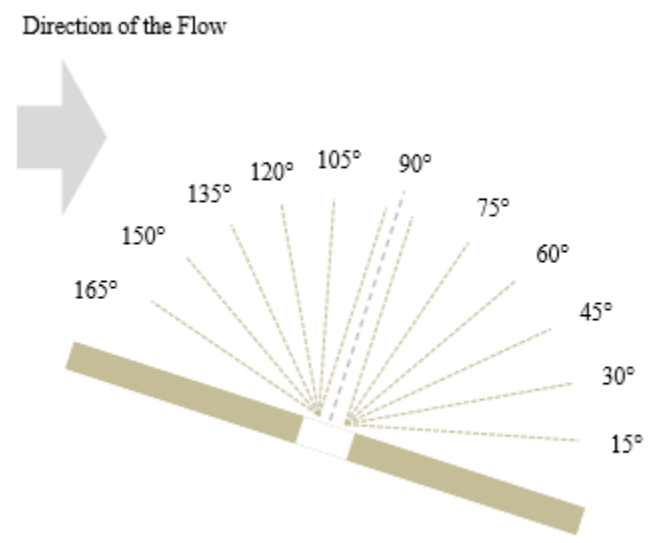

Fig. 3 Nomenclature for the inflow/outflow directions of the jet (note that the figure has been rotated $18^{\circ}$ to match the angle over the hump).

The jet velocity (in the prescribed inflow/outflow directions) has a parabolic profile across the jet slot and fluctuates in time, defined as

$$
V_{j e t}(\xi, t)=V_{j}(\xi) \cdot \sin \omega t
$$

where $\xi$ is the distance (across the jet slot) from the upstream end of the jet slot, $V_{j}(\xi)$ is a parabolic profile defined later in Section II.6, $\omega$ is the frequency $(100 \mathrm{~Hz})$, and $t$ is the time. The horizontal $(x)$ and vertical $(y)$ components of the velocity, considering the prescribed jet direction, are specified through a User Defined Function (UDF) in FLUENT. These velocity components across the jet exit are monitored throughout the simulation to assure that the jet direction and mass flow rate are as intended. For transient analyses, the time-step size is set at $5 \times 10^{-5}$ seconds (200 time-steps per jet oscillation cycle); this time-step size was found to be sufficiently small, i.e. the solution did not change with further reducing the time-step size.

For all simulations with a Synthetic Jet, we employ the following three steps:

1. Run a steady-state simulation for 15,000 iterations, which are sufficient for the scaled residual for continuity to decrease to $10^{-6}$.

2. Switch the simulation to the transient mode and run it for 6,000 time-steps (30 jet-oscillation periods) with 20 iterations per time-step to obtain statistically converged solutions. 
3. Run the simulation for another 2,000 time-steps (10 jet-oscillation periods) for taking time statistics, resulting in a total of 8,000 time-steps for each simulation (corresponding to about 33 flow-through times based on the hump chord).

\section{Grid convergence}

To achieve grid convergence, grids of different sizes were created and compared until the predictions of separation and reattachment points converged. The final two-dimensional mesh comprises 0.62 million elements, with the first 20 layers from the wall stretching at a growing rate of 1.1. The size of the smallest elements close to the hump surface is $0.03 \mathrm{~mm}$. Also, within $2 \mathrm{~mm}$ from the jet exit, there are two spherical zones with refined mesh elements of $0.02 \mathrm{~mm}$. (0.011 of the jet slot width; see Fig. 2). Table 2 shows the data obtained from three different grids with increasing the amount of elements in the vicinity of the wall, therefore reducing the $y^{+}$value.

Table 2 Summary of grid convergence study (without jet actuation).

\begin{tabular}{cccc}
$\begin{array}{c}\text { Number of } \\
\text { Elements }\end{array}$ & $\begin{array}{c}\text { Average } \\
y^{+}\end{array}$ & $\begin{array}{c}\text { Separation } \\
x / c\end{array}$ & $\begin{array}{c}\text { Reattachment } \\
x / c\end{array}$ \\
\hline Experiment & - & 0.665 & 1.11 \\
150,000 & 4.07 & 0.657 & 1.254 \\
620,000 & 0.82 & 0.655 & 1.232 \\
$1,500,000$ & 0.36 & 0.655 & 1.230
\end{tabular}

According to Karthikeyan et $\mathrm{al}^{18}$, for this type of RANS simulation, the solutions are fairly grid independent when the wall normal spacing is less than 2.5 wall units; moreover, grid refinement in the separated region does not improve the predictions. As can be seen from Table 2, once the $y^{+}$reaches a value close to one, very little changes are observed with further grid refinement. We use the mesh with 0.62 million elements for the rest of the study.

\section{Effect of jet exit velocity profile}

To confirm the effect of the velocity profile specified at the jet exit, we performed a set of preliminary simulations (for the BDSJ $165-15$ configuration, i.e. inflow and outflow angles are $165^{\circ}$ and $15^{\circ}$ ) with the S-A model, changing the profile from a top hat to a fully parabolic one. The profile is defined as:

$$
V_{j}(\xi)=V_{j, \max }\left(1-\left(\frac{\xi}{D}-0.5\right)^{2} S\right)
$$


where $V_{j, \max }$ is the maximum jet velocity at the middle of the jet slot, $D$ is the diameter (width) of the jet exit, and $S$ is a non-dimensional shape factor that defines the shape of the parabolic profile. Note that the value of $V_{j, \max }$ depends on $S$ to keep the mass flow rate constant. The results are summarized in Table 3. The separation and reattachment points were estimated from the location of the local minimum values of the mean friction coefficient over the hump/wall.

Table 3 Summary of preliminary simulations with different jet profiles (BDSJ 165-15 configuration).

\begin{tabular}{|c|c|c|c|c|c|c|}
\hline Case & $S$ & $V_{j, \max } / U_{\infty}$ & $\begin{array}{c}\text { Separation } \\
x / c\end{array}$ & $\begin{array}{c}\text { Reattachment } \\
x / c\end{array}$ & $\begin{array}{c}\text { Bubble length } \\
\qquad x_{b} / c\end{array}$ & $\begin{array}{c}\text { Difference in } \\
\text { bubble length } \\
\left(\text { Case }_{\mathrm{X}}-\text { Case }_{1}\right) \\
\text { /Case }_{1}\end{array}$ \\
\hline 1 & 0.0 & 0.86 & 0.7175 & 1.227 & 0.5095 & $0.00 \%$ \\
\hline 2 & 0.2 & 0.88 & 0.7175 & 1.228 & 0.5102 & $0.14 \%$ \\
\hline 3 & 0.6 & 0.91 & 0.7175 & 1.228 & 0.5104 & $0.18 \%$ \\
\hline 4 & 1.0 & 0.96 & 0.7175 & 1.229 & 0.5116 & $0.41 \%$ \\
\hline 5 & 2.0 & 1.03 & 0.7175 & 1.231 & 0.5133 & $0.74 \%$ \\
\hline 6 & 3.0 & 1.13 & 0.7175 & 1.230 & 0.5123 & $0.56 \%$ \\
\hline 7 & 4.0 & 1.29 & 0.7175 & 1.231 & 0.5132 & $0.73 \%$ \\
\hline
\end{tabular}

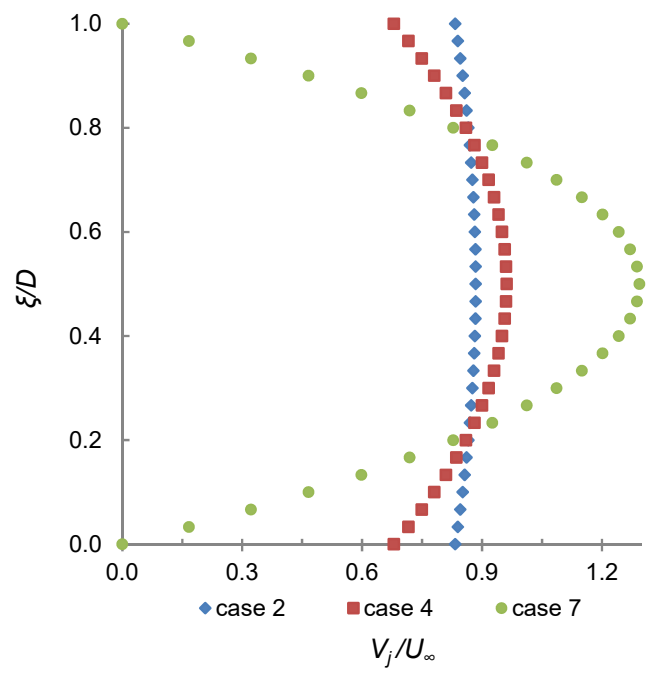

Fig. 4 Velocity profile at the jet exit for Cases 2, 4 and 7.

Fig. 4 shows the velocity profiles across the jet exit for Cases 2, 4 and 7. As can be seen from Table 3, the effects of different jet profiles on the separation bubble size are insignificant. Hence we use the jet velocity profile of Case 4 $\left(S=1\right.$ and $\left.V_{j, \text { max }} / U_{\infty}=0.96\right)$ for the rest of the study. 


\section{Model Validation}

Fig. 5 shows distributions of pressure coefficient $C_{p}$ over the hump with no flow control. Although a number of CFD results were compared with experiments in the 2004 workshop, only the 2D S-A results from Karthikeyan ${ }^{18}$ are shown in this figure as a reference. The reference CFD results, the experiments (PIV measurements ${ }^{27}$ ) and the current CFD results with the S-A model agree well at $x / c<0.65$ while at $x / c>0.65$ the results indicate some minor discrepancies. Also, a comparison of streamwise velocity at $x / c=0.8$ is presented in Fig. 6, again showing a good agreement.

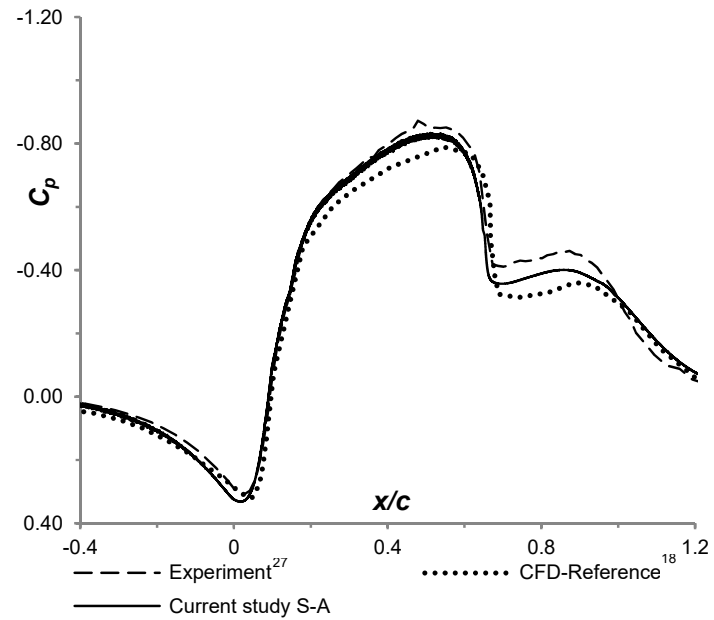

Fig. 5 Comparison of $C_{p}$ over the hump with no flow control.

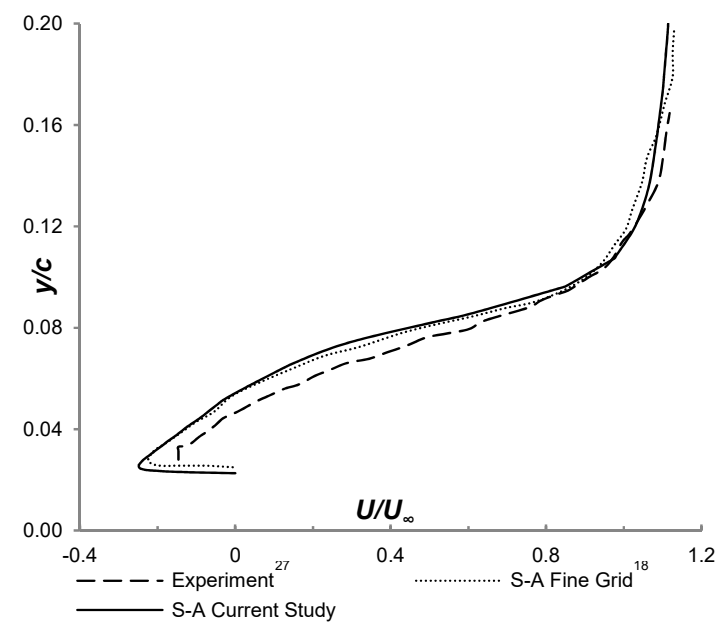

Fig. 6 Comparison of streamwise velocity at $x / c=0.8$ with no flow control.

Table 4 Separation and reattachment locations (classical Synthetic Jet configuration).

\begin{tabular}{|c|c|c|c|c|}
\hline Study & Author & $\begin{array}{c}\text { Separation } \\
x / c\end{array}$ & $\begin{array}{c}\text { Reattachment } \\
x / c\end{array}$ & $\begin{array}{c}\text { Bubble length } \\
x_{b} / c\end{array}$ \\
\hline Experiment & D. Greenblatt et al. ${ }^{27}$ & 0.680 & 0.980 & 0.300 \\
\hline DES & Saric et al. ${ }^{28}$ & 0.662 & 1.110 & 0.448 \\
\hline LES & Saric et al. ${ }^{28}$ & 0.671 & 1.050 & 0.379 \\
\hline RANS (average) & Rumsey et al. ${ }^{19},{ }^{29}$ & 0.670 & 1.220 & 0.550 \\
\hline$k-\omega \mathrm{SST}$ & & 0.713 & 1.345 & 0.632 \\
\hline S-A & Present study & 0.736 & 1.270 & 0.534 \\
\hline Realizable $k-\varepsilon$ & & 0.691 & 1.180 & 0.489 \\
\hline
\end{tabular}

Table 4 provides comparisons of the separation and reattachment locations for the classical Synthetic Jet case. These results indicate that the current CFD procedure can be used to predict the behavior of this type of flow 
qualitatively correctly; hence, similar procedures can be used to assess the qualitative performance of the novel Synthetic Jets configurations as well. It should be borne in mind that our objective is to assess the trend of the influence of different jet configurations and not the exact locations of the separation and reattachment.

\section{Results and discussion}

We performed a number of simulations, covering various combinations of inflow/outflow jet angles, with the three different turbulence models. Also, we employ three different approaches regarding the jet exit conditions in order to fully understand the characteristics of the Bi-Directional Synthetic Jet in comparison with the classical Synthetic Jet. These three approaches are: (i) Unfixed mass flow rate, i.e. keeping the same area (width) of the jet slot and the same amplitude of the velocity as the classical Synthetic Jet case; (ii) Unfixed velocity, i.e. keeping the same area (width) of the jet slot and the same mass flow rate as the classical Synthetic Jet case; and (iii) Unfixed jet area, i.e. keeping the same amplitude of the velocity and the same mass flow rate as the classical Synthetic Jet case. Note that it is impossible to fix these three parameters (mass flow rate, velocity and jet slot width) all together when we change the jet flow direction in this CFD study (since the jet exit boundary is always aligned with the hump surface, i.e. the jet flow direction is perpendicular to the jet exit boundary only when the jet flow direction is at $90^{\circ}$ in Fig. 3). For the "unfixed jet area" case, the middle point of the jet slot is fixed while changing the jet flow direction and the slot width, to make a fair comparison.

Fig. 7 shows comparisons of the separation bubble length between the most representative cases of the BDSJ with inlet-outlet jet directions of $165^{\circ}-15^{\circ}, 150^{\circ}-30^{\circ}, 135^{\circ}-45^{\circ}, 120^{\circ}-60^{\circ}, 105^{\circ}-75^{\circ}$, and the classical Synthetic Jet case $\left(90^{\circ}-90^{\circ}\right)$, for the three different approaches. The results for the three different turbulence models are all plotted for comparison, together with their average results. As can be seen from the figure, for all three different approaches, the general trend is that the BDSJ performs better than the classical Synthetic Jet, i.e. the separation bubble length reduces as we increase the difference between the inflow and outflow angles. The performance increase is most significant for the "unfixed velocity" case, where the amplitude of the jet velocity fluctuation increases as the inflow/outflow jet angles are changed (inclined) from $90^{\circ}$ (since the jet slot size and mass flow rate are fixed). It should be noted, however, that a higher jet velocity may result in a higher friction loss within the internal part of the Synthetic Jet and hence a higher power required to operate the device. 


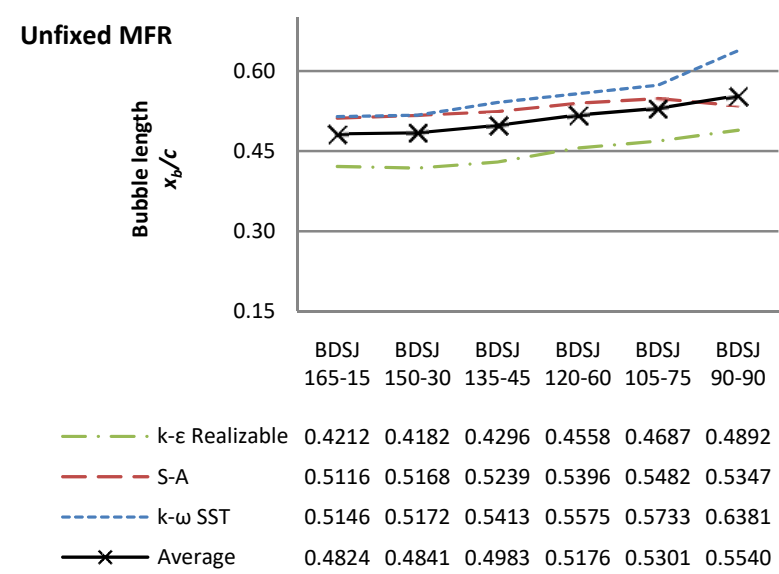

a)

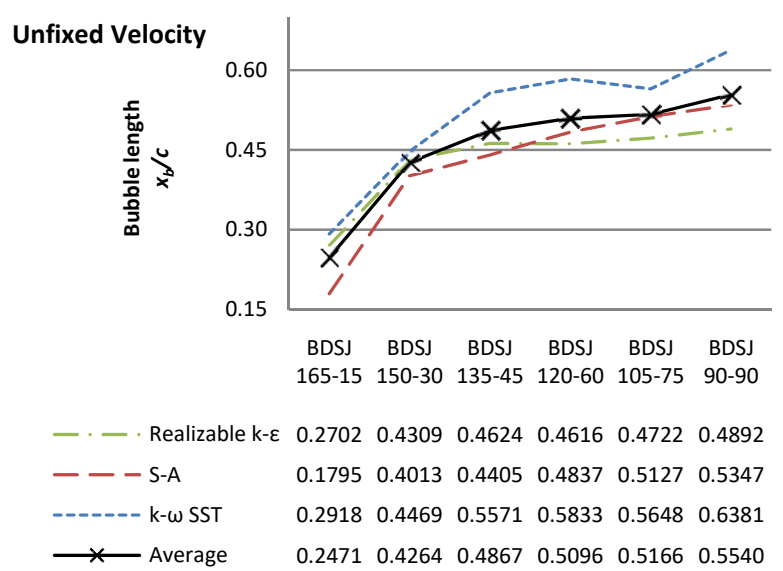

b)

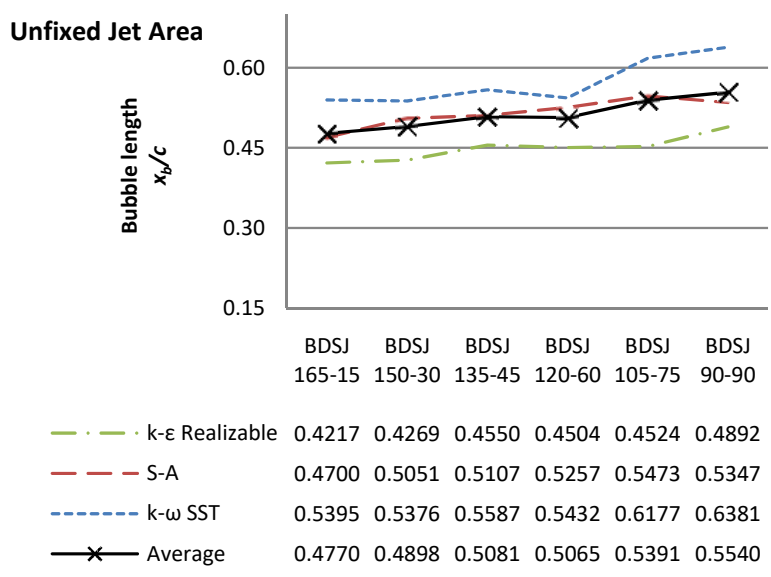

c)

Fig. 7 Comparison of the separation bubble length for most representative BDSJ cases: (a) Unfixed mass flow rate; (b) Unfixed velocity; (c) Unfixed jet area.

Fig. 8 shows comparisons of the contours of the time-averaged streamwise velocity over the hump for the cases with the classical Synthetic Jet and the BDSJ. These contours illustrate the effect of the different Synthetic Jet conditions on the size of the separation bubble not only in the streamwise but also in the wall-normal directions. 


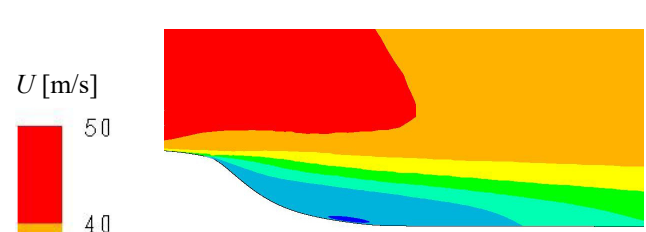

a) Synthetic Jet $-k-\varepsilon$

30

20

10

d) BDSJ-165-15 - $k-\varepsilon$ (Unfixed Area)

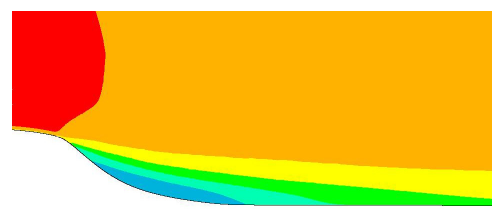

g) BDSJ-165-15 - $k-\varepsilon$ (Unfixed velocity)

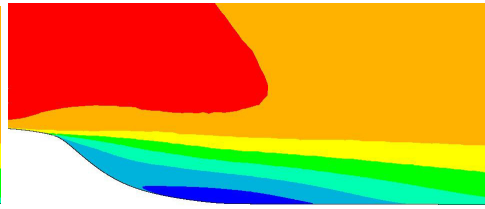

b) Synthetic Jet - S-A

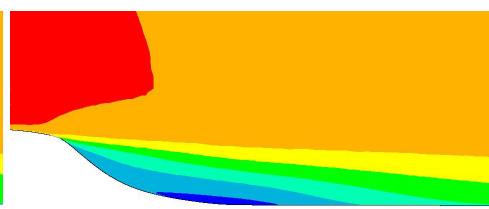

e) BDSJ-165-15 - S-A (Unfixed Area)

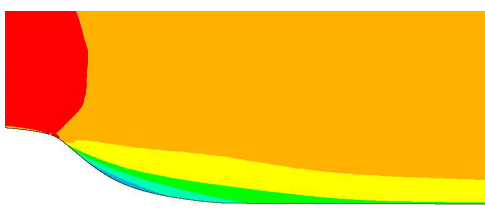

h) BDSJ-165-15 - S-A (Unfixed velocity)

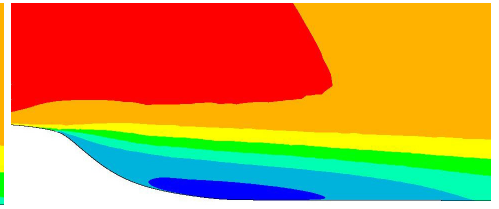

c) Synthetic Jet - SST

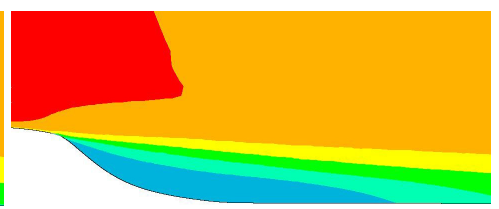

f) BDSJ-165-15-SST (Unfixed Area)

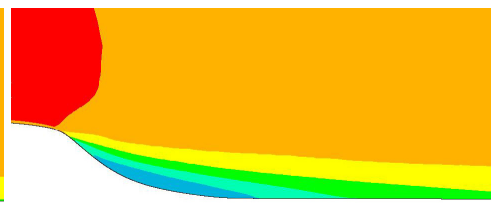

BDSJ-165-15 - SST (Unfixed velocity)

Fig. 8 Mean streamwise velocity contours above the hump.

\section{Conclusions}

In this study, we performed a set of 2D unsteady RANS simulations to examine potential benefits of changing the inflow and outflow directions of a Synthetic Jet, called the Bi-Directional Synthetic Jet (BDSJ). The simulations were performed for the so-called "NASA 2D Hump" configuration to assess the performance of the BDSJ compared to the classical Synthetic Jet. Three different turbulence models were employed to obtain general trends of the performance of the BDSJ. We also considered three different approaches regarding the jet exit conditions, namely the "unfixed mass flow rate", "unfixed velocity" and "unfixed jet area" cases, to make a meaningful comparison with the classical Synthetic Jet.

One of the key findings from this study is that the performance of a Synthetic Jet could improve significantly if the inflow (suction) and outflow (ejection) directions of the jet are optimized separately. The simulation results suggest that the performance of the BDSJ increases as we increase the difference between the inflow and outflow angles of the jet. The results also show that there is little influence of the different parabolic velocity profiles specified at the jet exit on the separation bubble size. All three turbulence models employed (S-A, SST and realizable $k-\varepsilon$ ) predict similar general trends. However, the results depend on the type of jet exit conditions 
employed; the jet performance improves most significantly when the jet mass flow rate and the slot width are fixed (and hence the jet velocity is unfixed) while changing the inflow/outflow directions of the jet.

It should be emphasized that the aim of the present study was to make an initial qualitative prediction of the performance of a hypothetical BDSJ device, on a widely used reference case (NASA 2D hump). This is a concept exploration study and we have not physically produced this device yet. Although it seems natural that the optimal outflow angle predicted is close to the direction tangential to the hump surface, further investigations using higher fidelity simulations, such as large-eddy and/or detached-eddy simulations, are required to fully understand the detailed flow mechanisms. It should also be noted that the optimal jet configuration may depend on the shape of the aerodynamic body and other flow parameters, such as the Reynolds number, freestream turbulence and the frequency and magnitude of the jet excitation.

\section{Acknowledgments}

The first author would like to acknowledge the National Council of Science and Technology of Mexico, CoNaCyT, for the financial support provided.

\section{References}

Glezer, A., and Amitay, M., "Synthetic Jets," Annual Rev. Fluid Mechanics, vol. 34, 2002, pp. 503-529.

Smith, B. L., and Glezer, A., "The formation and evolution of synthetic jets," Physics of Fluids, vol. 10, 1998, pp. 2281-2297.

Zhong, S., Millet, F., and Wood, N. J., "The behaviour of circular synthetic jets in a laminar boundary layer," The Aeronautical Journal, 2005, pp. 461-470.

Mallinson, S. G., Hong, G., and Reizes, J. A., "Some Characteristics of Synthetic Jets," AIAA Paper 99$3651,1999$.

Hong, G., "Effectiveness of micro synthetic jet actuator enhanced by flow instability in controlling laminar separation caused by adverse pressure gradient," Sensors and Actuators A: Physical, vol. 132, Nov. 2006, pp. 607-615.

Mohseni, K., and Mittal, R., Synthetic Jets: Fundamentals and Applications, CRC Press, Taylor \& Francis Group, 2015.

Lee, C. Y., and David B. Goldstein, "Two Dimensional Synthetic Jet Simulation,” AIAA Journal, vol. 40, 2002, pp. 510-516.

Rumsey, C., and Gatski, T., "Summary of the 2004 CFD Validation Workshop on Synthetic Jets and Turbulent Separation Control," 2nd AIAA Flow Control conferenceAIAA Flow Control conference, AIAA Paper 2004-2217, 2004.

Rumsey, C. L., "Successes and Challenges for Flow Control Simulations," AIAA Paper 2008-4311, 2008.

Gan, S., Ghia, U., and Ghia, K., "Active flow control of separated turbulent flow over a hump using RANS, DES and LES," FEDSM2008, ed., Jacksonville, Florida: ASME, 2008, pp. 523-530.

Greenblatt, D., Paschal, K. B., Yao, C.-S., Harris, J., Schaeffler, N. W., and Washburn, A. E., “A Separation Control CFD Validation Test Case Part 2 - Zero Efflux Oscillatory Blowing,” AIAA Paper 2005-0485, 2005. 
Rumsey, C. L., "Reynolds-Averaged Navier-Stokes Analysis of Zero Efflux Flow Control over a Hump Model," Journal of Aircraft, vol. 44, 2006, pp. 444-452.

Arwatz, G., Fono, I., and Seifert, A., "Suction and oscillatory blowing actuator Modeling and Validation," AIAA Journal, vol. 46, 2008, pp. 1107-1117.

Woszidlo, R., Nawroth, H., Raghu, S., and Wygnanski, I. J., "Parametric Study of Sweeping Jet Actuators for Separation Control," AIAA Paper 2010-4247, 2010.

Greenblatt, D., Paschal, K. B., Yao, C. S., Harris, J., Schaeffler, N. W., and Washburn, A. E., "Experimental Investigation of Separation Control Part 1: Baseline and Steady Suction," AIAA Journal, vol. 44, 2006, pp. $2820-2830$.

16 ANSYS Inc., ANSYS FLUENT 14 User's Guide, ANSYS, 2011.

17 Kawai, S., and Larsson, J., "Wall-modeling in large eddy simulation: Length scales, grid resolution, and accuracy," Physics of Fluids, vol. 24, 015105, 2012.

Rumsey, C. L., "Proceedings of the 2004 Workshop on CFD Validation of Synthetic Jets and Turbulent Separation Control," NASA/CP-2007-214874, 2007.

Rumsey, C. L., "Reynolds-Averaged Navier-Stokes Analysis of Zero Efflux Flow Control over a Hump Model," AIAA Journal, vol. 44, 2007, pp. 444-452.

Viken, S. A., Vatsa, V. N., Rumsey, L., and Carpenter, M. H., "Flow Control Analysis on the Hump Model with RANS Tools," AIAA Paper 2003-0218, 2003.

21 Laouedj, S., Azzi, A., and Benazza, A., "New Analysis in 3D-Synthetic Jets Within Numerical Investigation Using CFD Validation Code: Moving Boundary Techniques," Energy Procedia, vol. 19, 2012, pp. 226-238. Lyons, D. C., Peltier, L. J., Zajaczkowski, F. J., and Paterson, E. G., "Assessment of DES Models for Separated Flow From a Hump in a Turbulent Boundary Layer," Journal of Fluids Engineering, vol. 131, $111203,2009$.

Kim, W., Kim, S., Choi, K., and Kim, C., "Experimental and Computational study on flow characteristics by Synthetic Jets Configuration," AIAA Paper 2010-864, 2010.

He, C., Corke, T. C., and Dame, N., "Numerical and Experimental Analysis of Plasma Flow Control Over a Hump Model," AIAA paper 2007-0935, 2007.

Bettini, C., and Cravero, C., "Computational Analysis of Flow Separation Control for the Flow Over a WallMounted Hump Using a Synthetic Jet," AIAA Paper 2007-516, 2007.

Utturkar, Y., Mittal, R., Rampunggoon, P., and Cattafesta, L., "Sensitivity of synthetic jets to the design of the jet cavity," AIAA Paper 2002-0124, 2002.

Greenblatt, D., Paschal, K. B., Yao, C. S., and Harris, J., "Experimental Investigation of separation Control Part2: Zero Mass-Flux Oscillatory Blowing,” AIAA Journal, vol. 44, 2006, pp. 2831-2845.

Šarić, S., Jakirlić, S., Djugum, a., and Tropea, C., "Computational analysis of locally forced flow over a wall-mounted hump at high-Re number," International Journal of Heat and Fluid Flow, vol. 27, 2006, pp. 707-720.

Rumsey, C. L., Gatski, T. B., Sellers III, W. L., Vasta, V. N., and Viken, S. a., "Summary of the 2004 Computational Fluid Dynamics Validation Workshop on Synthetic Jets," AIAA Journal, vol. 44, 2006, pp. 194-207. 